\title{
Os efeitos da histerectomia sobre a sexualidade feminina
}

\author{
Amanda Albiero Real*, Maria Eduarda Parcianello Cabeleira*, \\ Juliana Rosa Nascimento*, Melissa Medeiros Braz ${ }^{* *}$, Hedionéia \\ Maria Foletto Pivetta***
}

Resumo: Objetivo - investigar a repercussão da histerectomia na sexualidade feminina. Metodologia - revisão integrativa de literatura em bases de dados eletrônicos LILACS, PubMED e CAPES, onde buscou-se periódicos publicados em língua portuguesa, espanhola e inglesa, entre os anos de 2002 a 2012, utilizando a associação dos descritores: "Histerectomia" e "Sexualidade", bem como seus equivalentes em inglês e espanhol. Resultados - Foram selecionados 17 artigos, os quais se relacionavam com o tema abordado. As disfunções sexuais relatadas pelos autores após a cirurgia foram diminuição de orgasmo, ausência de desejo e redução na frequência da atividade sexual. No entanto, outros estudos assinalam a melhora da qualidade de vida sexual após a histerectomia. Conclusão - a análise dos estudos encontrados demonstra grande divergência entre os achados. Sugere-se a realização de novas pesquisas que abordem a temática e busquem estabelecer estatisticamente essa correlação.

Descritores: Histerectomia, Sexualidade.

\section{The effects of hysterectomy on female sexuality}

Abstract: Objective - research in the literature articles that demonstrate the impact of hysterectomy on female sexuality. Methodology - bibliographic study on electronic databases LILACS, PubMED and CAPES, where were reached periodicals published in Portuguese, Spanish and English language, between 2002 and 2012, using the association of the descriptors: "Hysterectomy" and "Sexuality", as well as their equivalents in English and Spanish. Results - 17 articles were selected, which were related to the topic addressed. The sexual dysfunctions reported by the autors after surgery were: decreased orgasm, lack of desire, and reduction in frequency of sexual activity. However, other studies point to improvement on quality of sexual life after histerectomy. Conclusion - the analysis of the studies found shows great discrepancy between the findings. It is suggested the conduct of further researches on the theme and seek to establish this correlation statistically.

Descriptors: Hysterectomy, Sexuality.

\footnotetext{
*Acadêmica de Fisioterapia na Universidade Federal de Santa Maria (UFSM), Santa Maria, RS, Brasil.

**Fisioterapeuta pela Universidade do Estado de Santa Catarina (UDESC), Florianópolis, SC, Brasil. Doutora em Engenharia de Produção e Sistemas - Ergonomia pela Universidade Federal de Santa Catarina (UFSC), Florianópolis, SC, Brasil.

${ }^{* * * F i s i o t e r a p e u t a ~ p e l a ~ U n i v e r s i d a d e ~ F e d e r a l ~ d e ~ S a n t a ~ M a r i a ~(U F S M), ~ S a n t a ~ M a r i a, ~ R S, ~ B r a s i l . ~ D o u t o r a ~ e m ~ E d u c a c ̧ a ̃ o ~}$ pela mesma Instituição.
} 


\section{Introdução}

A histerectomia consiste na remoção cirúrgica do útero, e pode ser efetuada por via abdominal ou vaginal. No Brasil, a cada ano, cerca de 300 mil mulheres recebem a indicação de histerectomia e necessitam de cirurgia ${ }^{1}$. Segundo dados do DATASUS ${ }^{2}$, foram realizadas no país cerca de 62.565 histerectomias totais pelo Sistema Único de Saúde (SUS) em 2010. No ano de 2011, até o mês de junho, realizou-se 27.867 histerectomias no Brasil.

0 útero é um órgão biologicamente associado à reprodução e socialmente vinculado à feminilidade e sexualidade, por isso sua extirpação, além de constituir-se em ato agressivo e mutilante, interfere tanto na expressão da sexualidade feminina quanto na imagem corporal $\mathrm{e}$ vida social ${ }^{3}$. A retirada do útero pode causar prejuízos na qualidade da vida sexual da mulher, em suas condições emocionais e na qualidade do relacionamento estabelecido com 0 parceiro. A necessidade de realizar uma cirurgia para a retirada desse órgão pode, em muitos casos, provocar emoções conflitivas, traumáticas, de inseาgurança e ansiedade, gerando mudanças importantes nos padrões e nos desejos sexuais 4 .

A histerectomia é uma cirurgia irreversível, que leva a modificação da integridade corporal e está impregnada de simbolismos. Mulheres submetidas á esse procedimento podem apresentar alterações na autoimagem e sintomas depressivos ${ }^{5}$, devido a concepções acerca do útero que estão intimamente atreladas à busca por exercer controle sobre a sexualidade da mulher, utilizando como justificativa a necessidade de preservá-lo para a maternidade, que foi vista por muito tempo como a única área em que as mulheres tinham uma missão social - a reprodução ${ }^{6}$.

Por outro lado, alguns homens têm medo de ferir suas mulheres durante 0 ato sexual, uma vez que elas não têm mais o órgão. Aparecem várias fantasias no período préoperatório, contribuindo para que as mulheres sintam-se abaladas quanto à "condição de ser mulher", uma vez que lhes foi retirado o útero ${ }^{3}$.

Além dos aspectos emocionais, ocorrem também modificações anatômicas na pelve, que poาdem levar a alteração do tamanho e/ou do formato dos órgãos genitais, dificuldade de penetração vaginal, dispareunia, interrupção dos suportes anatômicos da resposta sexual, rebaixamento do impulso sexual e do grau de atratividade por redução de níveis hormonais circulantes, decorrentes de alterações circulatórias, ocasionando, em última instância, disfunções sexuais. Autores relatam que a histerectomia pode causar encurtamento da vagina, diminuição da libido e menor frequência de orgasmos após a penetração ${ }^{7}$.

Nesse intuito, coloca-se como questão a ser investigada: quais as repercussões da histerectomia sobre a sexualidade feminina?

Com isso, o objetivo traçado foi buscar na literatura estudos que sinalizem a repercussão da histerectomia na sexualidade da mulher em idade reprodutiva.

Saúde (Santa Maria), v.38, n.2, p. 123-130, 2012. Os efeitos da histerectomia sobre a sexualidade

feminina

\section{Metodologia}

Este estudo foi elaborado a partir de uma revisão integrativa em periódicos de bases de dados eletrônicas no período de 2002 a 2012. O estudo seguiu as seguintes etapas: escolha da temática e delineamento do problema de pesquisa, definição dos critérios de inclusão/exclusão, busca em sites eletrônicos, seleção dos estudos, análise, síntese e interpretação dos mesmos. 
A pesquisa pelos artigos foi realizada nos periódicos da CAPES, LILACS e PUBMED através da associação dos descritores: "Histerectomia" e "Sexualidade", bem como suas equivalências em inglês e espanhol.

Os critérios de inclusão utilizados foram: periódicos escritos em português, inglês ou espanhol, publicados entre os anos de 2002 e 2012 que relacionassem alterações na sexualidade feminina com a cirurgia de histerectomia. Os estudos que descreviam apenas a técnica cirúrgica ou disfunções sexuais sem a realização de cirurgia foram excluídos. Também foram descartadas revisões bibliográficas, capítulos de livros, teses, dissertações, trabalhos de conclusão de curso, resumos e anais de eventos científicos, pois foi elencada a busca por estudos científicos publicados em periódicos, disponíveis nas bases de dados já anunciadas.

A análise dos dados foi realizada mediante a leitura minuciosa dos artigos selecionados, para posterior sintetização e interpretação dos dados mais relevantes. Os resultados são apresentados descritivamente.

\section{Resultados}

Foram encontrados 220 artigos, classificados conforme os descritores, entretanto, de acordo com os critérios de inclusão e exclusão foram selecionados 17 , relacionados com a temática abordada, como demonstra a tabela que segue. Dos artigos selecionados, 08 eram em língua inglesa, 07 eram em língua portuguesa e 02 em língua espanhola.

Tabela 1 - Número de artigos encontrados de acordo com os descritores histerectomia e sexualidade nas bases de dados CAPES, LILACS e PUBMED, em 2012.

\begin{tabular}{ccc}
\hline Descritores/Bases de dados & Artigos encontrados & Artigos selecionados \\
\hline CAPES & 55 & 07 \\
LILACS & 21 & 05 \\
PUBMED & 144 & 05 \\
Total de artigos & 220 & 17 \\
\hline
\end{tabular}

\section{Discussão}

A intervenção cirúrgica ginecológica é algo que denota uma invasão ao corpo feminino. Implica a modificação da estrutura corporal, e mais do que isto, a manipulação de partes do corpo relacionadas à sexualidade e identidade feminina, podendo acarretar modificações profundas na imagem corporal ${ }^{8}$.

É essencial que o profissional lance um olhar extensivo sobre a sexualidade. $O$ ato sexual compreende apenas uma face da sexualidade. A sexualidade de uma mulher está na forma como ela se expressa em sua vida, em seu meio; dá o tom, a sua maneira de conduzir a sua vida ${ }^{9}$.

Saúde (Santa Maria), v.38, n.2, p. 123-130, 2012 Real, A. A., et al. 
De fato, os efeitos da histerectomia na vida da mulher e, mais especificamente, na sexualidade feminina, são complexos e decorrentes da interação de fatores físicos, psicológicos, sociais, culturais, religiosos e educacionais, que interferem na visão que a mulher tem do útero e de si mesma. Essa mudança é dependente de crenças e valores que esta mulher possui, relacionados ao útero e ao gênero feminino. Essas crenças e valores, por sua vez, são oriundos da interação da mulher com uma sociedade marcada pelo sistema patriarcal que, valorizando a maternidade como aspecto essencial em sua vida, confere ao útero um sentido de fornecedor de identidade à mulher, sendo necessário à confirmação de sua feminilidade ${ }^{9}$.

Diante da notícia de que terá este órgão extirpado, a mulher passa minimamente por dois tipos de problemas: o medo da cirurgia propriamente dita e da mutilação de um órgão que representa a maternidade e de certa forma a sexualidade feminina ${ }^{10}$.

A retirada do útero frequentemente traz anseios e questionamentos, baseados em crenças e valores que podem induzir a reformulações ligadas ao feminino, à representação social, ao gênero, à autoimagem, à sexualidade e à relação conjugal e social ${ }^{8}$.

A preocupação de ser fértil, ter a viabilidade de gerar vidas é geralmente uma constante cobrança da sociedade e, muitas vezes, da própria mulher. $O$ útero, então, é o que confere a representação como mulher e a possibilidade do estatuto social de mãe. As repercussões da histerectomia dependem da idade da mulher, do desejo ou não de futuras gestações, da reação do companheiro, da escolha ou não de submeter-se ao procedimento e da análise dos benefícios ou malefícios da retirada do útero. Para algumas mulheres, a histerectomia tem uma conotação de cura, alívio, de resolução de problemas, e para outras, a cirurgia traz muita insegurança ${ }^{8}$.

As mulheres a serem submetidas à histerectomia podem apresentar comprometimento na sexualidade, reprodução e função sexual. Para elas, a histerectomia poderia ser o evento que, de fato, encerraria o período reprodutivo de sua vida com grandes comprometimentos na sua sexualidade. Mais da metade das mulheres apresenta incertezas em relação à retira $\mathrm{nda}$ do útero e sobre o real impacto deste procedimento sobre a vida sexual, assim como também reportam sentimentos de tristeza por perderem um órgão importante contraposto ao desejo de cura ${ }^{11}$.

A maioria das mulheres considera a cirurgia como um evento negativo, ancorando as vivências pós-operatórias na impossibilidade de se tornarem mães, na incapacidade de manterem o casamento, nas incertezas quanto à capacidade de sentirem prazer e de serem socialmente aceitas. Mas para outras, a histerectomia é uma forma de resgatar a vida social, uma vez que lhes possibilita reconquistar a liberdade de sair, passear e viajar, interferindo positivamente no processo de viver e na qualidade de vida ${ }^{12}$.

Quanto ao relacionamento sexual após a histerectomia, 39,4\% das pacientes exibem piora; $33,3 \%$ melhora e $27,3 \%$ relatam que a cirurgia não exerce impacto positivo ou negativo sobre a sexualidade. A histerectomia parece prejudicar a vida sexual e a sexualidade das mulheres, pois o útero está diretamente associado a estas funções. Os escores médios obtidos após a aplicação de questionário mostram piora significante nos parâmetros "satisfação sexual"; "expressão da sensualidade feminina" e "anorgasmia"11.

Acredita-se que mulheres submetidas à histerectomia podem apresentar alterações no Saúde (Santa Maria), v.38, n.2, p. 123-130, 2012.
Os efeitos da histerectomia sobre a sexualidade autoconceito, sintomas depressivos e psicossomáticos, dificuldade de relacionamento sexual e conjugal, devido a associações psicológicas feitas entre a procriação e a sexualidade ${ }^{11}$. No entanto, as reais consequências da realização da histerectomia sobre a qualidade da vida 
sexual posterior à cirurgia são controversas e dependem diretamente da intensidade dos sintomas pré-cirúrgicos, das condições emocionais e da qualidade do relacionamento estabelecido com o parceiro. Estudos não apontam, no entanto, de forma conclusiva, os efeitos pós-cirúrgicos sobre a qualidade de vida sexual da mulher, deixando lacunas acerca da real interferência emocional do procedimento ${ }^{13,3,5}$.

Entre 352 pacientes submetidas à histerectomia que responderam um questionário sobre sua vida sexual após a cirurgia, 310 relataram ser sexualmente ativas antes e depois da realização da mesma. Dez pacientes que foram sexualmente ativas antes da cirurgia já não eram sexualmente ativas seis meses após a cirurgia. Das 32 pacientes que não eram sexualmente ativas antes da histerectomia, 17 se tornaram após a cirurgia. Esse mesmo estudo concluiu que para a grande maioria das mulheres submetidas a algum tipo de histerectomia, o bem-estar sexual melhora após a cirurgia ${ }^{13}$.

A histerectomia pode representar a descoberta de outros poderes e potencialidades que podem significar a liberdade, o autoconhecimento e uma grande fonte de energia e prazer genital ${ }^{9}$.

A melhora na função sexual após essa cirurgia pode indiretamente estar associada com as consequências da mesma, como menos preocupação sobre a gravidez indesejada, ausência de sangramento vaginal e mais tempo para atividades sexuais pela cessação da menstruação ${ }^{14}$. A maioria dos relatórios sobre o funcionamento sexual após histerectomia encontraram benefícios significativos em vários aspectos do funcionamento sexual ${ }^{15}$. Porém, há estudos que se contrapõem a essa ideia, mostrando que a melhoria na função sexual pós histerectomia, mesmo aos 24 meses de acompanhamento, não é estatisticamente significativa ${ }^{16}$.

Em estudo realizado no Chile, em relação à qualidade da vida sexual após histerectomia, a grande maioria das 90 mulheres participantes da pesquisa apontou melhora após 6 meses de cirurgia ${ }^{17}$.

Estudos prospectivos têm sugerido que a maioria das mulheres pode ter sua função sexual inalterada ou melhorada após a histerectomia ${ }^{18,19}$. No entanto, uma minoria substancial de mulheres relatam os resultados adversos da função sexual após a cirurgia ${ }^{19}$.

A histerectomia pode melhorar significativamente a satisfação sexual em itens como excitação, frequência de orgasmo e desejo pelo parceiro ${ }^{20}$. Mulheres submetidas à histerectomia abdominal retomam sua vida sexual mais tardiamente comparado àquelas que realizam cirurgia vaginal ou laparoscópica. A qualidade da relação sexual é considerada inalterada $(60,4 \%)$, melhor $(21,3 \%)$ e bem pior em $18,3 \%$ dos pacientes. No entanto, deterioração da função sexual é mais frequente no grupo histerectomia abdominal $(24 \%)$ do que nos outros dois tipos de histerectomia ${ }^{21}$.

As mulheres atribuem ao útero à função de gerar filhos. Logo, se o útero não servia mais para gerar filhos, ele também seria desprovido de significado para elas. As mulheres, portanto, apresentam apenas uma definição funcionalista acerca do útero, que se reflete na maneira como percebem a si próprias também como seres sociais. Nesse momento, a retirada do útero é vista como a perda de suas próprias funções, o que pode facilitar a assimilação de mitos que discorrem sobre as mulheres ficarem vazias, "ocas" depois da saúde (santa Maraia, v38, n.2. p. 123.130, 2012. histerectomia. Ao mesmo tempo, a iminência da histerectomia suscita uma série de reelaborações na autoimagem das mulheres, no sentido de buscarem uma reconfiguração de sua identidade social ${ }^{3}$. 
No que se trata de características da sexualidade e sua evolução em mulheres histerectomizadas, não se encontra diferenças significativas na frequência de relações sexuais, presença e frequência de orgasmo, percepção de desejo sexual e grau de excitação sexual, concluindo que a histerectomia não afeta negativamente a sexualidade, salientando a importância da educação sexual para essas mulheres ${ }^{22}$.

É essencial disponibilizar um espaço de problematização e escuta acerca do viver sem útero, da feminilidade e sexualidade com vistas a prevenir conflitos pessoais e conjugais entre as mulheres em processo de histerectomia ${ }^{12}$. É necessário que o profissional aborde a retirada do útero a partir de uma perspectiva de atenção integral à saúde das mulheres e no contexto de humanização do atendimento, onde os mitos sejam confrontados com a informação científica, de maneira que Ihes seja permitido vivenciar a cirurgia e o pósoperatório do modo menos inquietante e mais satisfatório. ${ }^{3}$ Acredita-se que um atendimento psicológico e um ambiente hospitalar favorável sejam imprescindíveis para que as mulheres possam vivenciar e elaborar as repercussões da retirada do útero de maneira menos prejudicial possível ${ }^{11}$.

\section{Conclusão}

A análise dos estudos encontrados relaciona o útero à representação da sensualidade e sexualidade feminina, vinculada à questão da reprodução. A histerectomia, por sua vez, acarreta modificações na estrutura corporal, tanto de ordem anatômica quanto funcional. Entretanto, a abordagem encontrada nos estudos investigados reporta a histerectomia aos aspectos de ordem psicoemocional e cultural acerca dessa representação do órgão.

Autores afirmam que a histerectomia implicou em alterações na vida sexual, decorrentes de repercussões negativas sobre 0 autoconceito e a autoestima. As disfunções sexuais relatadas pelas mulheres foram diminuição de orgasmo, ausência de desejo pelo parceiro e redução na frequência da atividade sexual.

Outros autores assinalam a melhora da qualidade de vida sexual após a histerectomia, que pode representar liberdade e fonte de prazer sexual, aumentando o desejo, frequência de orgasmo e atração do parceiro.

A partir desses resultados contraditórios, é importante ressaltar que as razões que defendem a piora ou melhora da vida sexual pós-histerectomia remetem a conotações psicossociais.

Como são encontradas pesquisas que acenam para as repercussões negativas e outras para a possibilidade de melhora na vida sexual, sugere-se a realização de outros estudos que abordem a temática e busquem estabelecer estatisticamente essa correlação.

Saúde (Santa Maria), v.38, n.2, p. 123-130, 2012. 


\section{Referências}

1. Sbroggio AMR. A ausência do útero associada ao conceito de feminilidade. Siicsalud. [on-line]. [citado 16 maio 2008]. Disponivel em: <http://www.siicsalud.com/dato/experto.php/86408>

2. DATASUS (Departamento de Informática do SUS), Sistema de Informações Hospitalares do SUS (SIH/ SUS). Disponivel em:<http://www.datasus.gov.br>. Acesso em: 22/08/ 2012.

3. Sbroggio AMR, Osis MJMD, Bedone AJ. O significado da retirada do útero para as mulheres: um estudo qualitativo. Rev Assoc Med Bras. 2005; 51(5): 270-274.

4. Naughton MJ, Mcbee WL. Health-related quality of life after hysterectomy. Clin Obstet Gynecol. 1997; 40(4): 947-957.

5. Goetsch MF. The effect of total hysterectomy on specific sexual sensations. Am J Obstet Gynecol. 2005; 192(6): 1922-1927.

6. Heilborn ML. Gênero: uma breve introdução. In: Ribeiro NMGR, Costa DM, organizadores. Gênero e desenvolvimento institucional em ONGs. Rio de Janeiro: IBAM/ENSUR/NEMPP. 1995; 9-14.

7. Zobbe V, Gimbel H, Andersen BM, Filtenborg T, Jakobsen K, Sorensen HC, et al. Sexuality after total vs. subtotal hysterectomy. Acta Obstet Gynecol Scand. 2004; 83(2): 191-196.

8. Silva CMC, Santos IMM, Vargens OMC. A repercussão da histerectomia na vida de mulheres em idade reprodutiva. Esc Anna Nery Rev Enferm. 2010; 14(1): 76-82.

9. Salvador RT, Vargens OMC, Progianti JM. Sexualidade e histerectomia: mitos e realidade. Rev Gaucha Enferm. 2008; 29(2): 320-323.

10. Araujo TVB, Aquino EML. Fatores de risco para histerectomia em mulheres brasileiras. Cad. Saúde Pública. 2003; 19(2): 407-417.

11. Tozo IM, Moraes JC, Lima SMR, Gonçalves N, Auge APF, Rossi LM, et al. Avaliação da sexualidade em mulheres submetidas à histerectomia para tratamento do leiomioma uterino. Rev Bras Ginecol Obstet. 2009; 31(10): 503-507.

12. Nunes MPRS, Gomes VLO, Padilha MI, Gomes GC, Fonseca AD. Representações de mulheres sobre a histerectomia. Esc Anna Nery Rev Enferm. 2009; 13(3): 574-581.

13. Roovers JP, Van Der Bom JG, Van Der Vaart CH, Heintz AP. Hysterectomy and sexual wellbeing: prospective observational study of vaginal hysterectomy, subtotal abdominal hysterectomy, and total abdominal hysterectomy. BMJ. 2003; 327, 7418: 744-748.

14. Maas CP, Weijenborg PT, Kuile MM. The effect of hysterectomy on sexual functioning. Annu Rev Sex Res. 2003; 14: 83-113.

15. Garry R, Fountain J, Brown J, Manca A, Mason S, Sculpher M, et al. Evaluate hysterectomy trial: a multicentre randomised trial comparing abdominal, vaginal and laparoscopic methods of hysterectomy. Health Technol Assess. 2004; 8(26): 151-154.

16. Wouter JKH, Volkers NA, Wouter B, Blok S, Birnie E, Reekers JA, et Al. Sexuality and Body Image After Uterine Artery Embolization and Hysterectomy in the Treatment of Uterine Fibroids: A Randomized Comparison. Cardiovasc Intervent Radiol. 2007; 30(5): 866-875.

17. Urrutia MT, Araya A, Rivera S, Viviani P, Villarroel L. Sexualidad de la mujer histerectomizada: modelo predictivo al seto mes posterior a la cirugía. Rev Med Chile. Chile, 2007; 135(3): 317-325.

18. Thakar R, Ayer S, Staton S, Manyonda I. Outcomes after total versus subtotal abdominal hysterectomy. N Engl J Med. 2002; 24(17): 1318-1325.

Saúde (Santa Maria), v.38, n.2, p. 123-130, 2012 Real, A. A., et al.

19. Flory N, Bissonette F, Binik YM. Psychosocial effects of hysterectomy: literature review. J Psychosom Res. 2005; 59: 117-129. 
20. Aziza A, Bergquista C, Nordholm L, Moller A, Silfverstolpe G. Prophylactic oophorectomy at elective hysterectomy Effects on psychological well-being at 1-year follow-up and its correlations to sexuality. Maturitas. 2005; 51: 349-357.

21. Ayoub JM, Fanchin R, Monrozies X, Imbert P, Reme JM, Pons JC. Respective consequences of abdominal, vaginal, and laparoscopic hysterectomies on women's sexuality. Eur J Obstet Gynecol Reprod Biol. 2003; 111: 179-182.

22. Urrutia MTS, Araya AG, Villarroel LDP, Viñales DA. Características y evolución de la sexualidade em mujeres histerectomizadas. Rev Chil Obstet Ginecol. 2004; 69(4): 301-306004.

\section{Amanda Albiero Real}

Endereço para correspondência - Rua dos Andradas, 777, casa 207. Bairro: Passo d'Areia. Santa Maria-RS, Brasil. CEP: 97010031.

E-mail: amanda.albiero@hotmail.com

Currículo Lattes: http://lattes.cnpq.br/8547169416465628

Recebido em 25 de setembro de 2012.

Aprovado em 22 de novembro de 2012. 